\title{
A inteligência competitiva e a área de informação tecnológica no Instituto de Pesquisas Tecnológicas do Estado de São Paulo S.A.
}

\section{Sonia Maria Akiko Wada Tomimori}

\section{Resumo \\ O artigo apresenta uma avaliação da mudança de paradigma que está sendo implementada na atual gestão do Centro de Informação Tecnológica (Citec), do Instituto de Pesquisas Tecnológicas do Estado de São Paulo (IPT-SP). \\ Busca-se demonstrar a atuação do profissional de informação como agente de mudança e as formas como pode intervir na cultura da organização, dentro da nova estratégia do IPT, de compartilhamento dos riscos e benefícios do desenvolvimento tecnológico entre os setores produtivo, privado e público.}

\section{Palavras-chave}

Informação tecnológica; Atuação do profissional da informação; O professional como agente de mudanças; Cultura da organização.
O atual projeto para o desenvolvimento da área de informação tecnológica no IPT foi decorrente de sua evolução, desde a sua fundação até as constantes mudanças ocorridas nas últimas décadas, no setor de teleinformática e na área de informação científica e tecnológica, no país.

O IPT, atento às principais demandas tecnológicas do país, tem convicção de que, na era da competitividade com qualidade, cabe a uma instituição de tal porte cumprir um papel específico junto às forças de mercado. A nova estratégia de atuação, desse modo, passa a ser o compartilhamento, com os setores produtivo, privado e públi$\mathrm{co}$, dos riscos e benefícios do desenvolvimento tecnológico.

Uma avaliação da mudança de paradigma que está sendo implementada na atual gestão do Centro de Informação Tecnológica (Citec) será aqui apresentada buscando demonstrar a atuação do profissional da área de informação como agente de mudança e sua intervenção na cultura da organização, com reflexos em seu ambiente interno e externo.

A informação, fator tão importante para as empresas quanto os recursos humanos, financeiros e materiais de que elas dispõem, tem constituído para os administradores do Citec/IPT, órgão prestador de serviços de informação científica e tecnológica, uma preocupação constante. Fatores como pertinência, oportunidade, precisão da informação e seu uso otimizado são ali gerenciados no sentido de contribuir diretamente para o incremento de pesquisas, apropriação de novas tecnologias e processos de inovação por parte do setor produtivo nacional.

Para tornar a informação científica e tecnológica disponível e adequada ao desenvolvimento de um projeto, é necessário ter capacitação quanto à orientação técnica, de forma precisa, com a redução do tempo de execução e, conseqüentemente, a otimização de seus custos. Nesse sentido, o Citec/IPT tem se preocupado em adquirir, manter e divulgar informações pertinentes e contextualizadas para facilitar o processo de comunicação e contribuir para que as empresas estejam informadas sobre tudo o que está sendo desenvolvido no mundo em sua área específica de atuação. Além disso, como não poderia deixar de ser, o Citec/IPT tem se preocupado, também, com a disseminação dessas informações por meio de telemática, área em que o país está sofrendo grandes e profundas transformações no momento.

O público a que se destina os serviços do Citec/IPT são investidores, corretoras, administradoras de fundos e consultorias de informação de negócios, indústrias e empresas que necessitam de informações tecnológicas, econômicas e estratégicas, para subsidiar a tomada de decisão para competirem em uma economia de mercado globalizado. Certamente, estes segmentos estão dispostos a comprar informações com valor agregado, voltadas ao atendimento de necessidades dessa natureza. 
Para o atendimento às prerrogativas para a inserção do país em um mercado globalizado, percebe-se a necessidade de criar mecanismos para adequar os serviços prestados pelo Citec/IPT a essas novas visões e exigências.

Nesse cenário, torna-se vital que os profissionais que trabalham com a prestação de serviços de informação reciclem seus conhecimentos e adaptem seus perfis de competências e habilidades às novas necessidades que se apresentam.

É neste contexto que se desenvolveu e consolidou o setor de informação tecnológica no IPT. O Citec, hoje, está concretizando sua reestruturação, transformando-se de centro de documentação tradicional, para centro de excelência em informação tecnológica.

O Citec/IPT encontra-se atualmente em fase de racionalização e modernização de suas formas de gestão, como também está buscando sua auto-sustentação com a prestação de serviços de informação. Este novo direcionamento foi decorrente de uma mudança tanto na conduta da alta direção do IPT, quanto na evolução pessoal, natural em um envolvimento constante com ambiente em transformação. Além desses fatores, vale ressaltar que o momento é de fato propício para isso, pois decorre não só do amadurecimento ao longo de uma trajetória profissional, mas também da incorporação em nossa equipe de trabalho de profissionais com experiência em inteligência competitiva para responder à crescente demanda de informação para atendimento ao setor industrial. O comprometimento do gestor da informação em estar habilitado para usar metodologias modernas e eficientes capazes de agregar valor à informação estratégica demandada pelo mercado exige uma mudança na postura e, conseqüentemente, na cultura organizacional do Citec, o que vem ocorrendo, sutilmente e de forma sistemática, no sentido de se adotarem novas formas de gestão da informação. Esta mudança tem levado em consideração, características tais como quantidade, oportunidade, conteúdo, qualidade, disponibilidade e organização da informação, conside- rando-se a sua utilização em tempo real como subsídio à execução de projetos de desenvolvimento tecnológico com informações necessárias à agilização e à segurança de processos decisórios.

A preocupação com a sobrevivência da área de informação no IPT gerou a necessidade de prepará-la para concorrer no mercado e, em decorrência, o modelo de gestão atual está se deslocando da visão centrada na coleção para uma organização centrada no acesso. Para tanto, houve necessidade de trabalhar com especialistas de diferentes áreas do conhecimento, além de estar sendo estimulado o trabalho em equipe, o que requer maior responsabilidade individual e autodisciplina nas relações interpessoais e hierárquicas.

A ênfase é dada no atendimento ao cliente com qualidade, com maior eficiência e eficácia, às demandas internas (pesquisadores e técnicos do IPT) e externas (comunidade científica e tecnológica) do mercado produtivo. Esta nova postura exige a adoção de sistema de gestão que permita ao Citec estar próximo ao mercado, ou seja, contribuir na exploração inteligente das fontes e na rentabilização de produtos e serviços oferecidos. Neste processo, destaca-se a introdução do conceito de desenvolvimento de metodologias e de novos serviços informacionais à luz das necessidades dos usuários, a valorização e proteção da informação tecnológica produzida no IPT e a implementação de modernas tecnologias (software e hardware) de tratamento, acesso, recuperação e gestão da informação.

Na década de 90, o contexto de sistemas informacionais de ambiente tecnológico no Brasil apresenta-se favorável do ponto de vista da infra-estrutura, notadamente no que diz respeito às mudanças nas formas de gestão da informação. A política nacional de tecnologias, serviços e indústria da informação teve grande impulso nos últimos anos, com a definição, pelos ministérios da Ciência e Tecnologia e das Comunicações, de regras para exploração de serviços Internet, bem como da implantação, no país, da Rede Nacional de Pesquisa (RNP) pelo Ministério da Ciência e Tecnologia, cobrindo todas as capitais do país.

O IPT, acompanhando esta tendência, modernizou e racionalizou o Citec para oferecer serviços e produtos de informação adequados e eficazes aos seus clientes, e o conhecimento do cliente e adequação dos serviços para a solução de seus problemas passam a constituir a postura fundamental na prestação de serviços de informação para o setor produtivo.

Em 1995, foi decidido que haveria necessidade de centralizar, do ponto de vista organizacional e físico, na Coordenadoria de Informação e Documentação (CID), as 12 bibliotecas especializadas existentes nas unidades técnicas do IPT localizadas no Campus da USP, em São Paulo, com exceção de uma na cidade de Franca.

O maior problema enfrentado foi a autonomia e independência das bibliotecas e sua subordinação às diretorias das unidades técnicas. Além das características próprias e peculiares de cada uma, outro problema enfrentado foi o de que a maioria dos pesquisadores e dos diretores das unidades técnicas não estava a favor dessa centralização.

Houve então considerável racionalização no que se refere à questão do quadro de pessoal. No início de 1994, a área de informação e documentação do IPT contava com 103 colaboradores passando para 76 colaboradores, sendo que 49 do quadro de pessoal e 27 estagiários, composta por profissionais de nível superior, sendo 19 bibliotecários (56\%) e de15 profissionais de outras áreas (44\%).

A partir de 1996, a CID é transformada em Centro de Informação Tecnológica (Citec) e conta, atualmente, com uma equipe de 36 colaboradores, sendo 15 bibliotecários (42\%) e de 21 profissionais de outras áreas de nível universitário e médio (58\%).

Outro aspecto importante dessa mudança foi a integração dos acervos e serviços de informação oferecidos pelas diferentes áreas do IPT. A partir dessa nova visão, a diversificação e adequação dos serviços às novas 
realidades são fatores de fundamental importância para a sobrevivência da área como um todo.

\section{ESTRATÉGIAS ADMINISTRATIVAS DE ATUAÇÃO DO CITEC/IPT}

A missão do Citec, órgão de informação tecnológica do IPT, com o novo enfoque institucional, tem por objetivos:

- atender à sua clientela interna, bem como ao setor produtivo público e privado, com o desenvolvimento e a oferta de serviços e produtos informacionais;

- organizar, sistematizar, assegurar a proteção e a valorização da informação tecnológica produzida no IPT, assim como do importante acervo de livros, periódicos, normas técnicas e catálogos de produtos industriais, além de acesso a bases de dados nacionais e internacionais;

- responder pela memória técnica do IPT, mantendo e organizando todos os registros da produção técnica do Instituto;

- participar de Redes Nacionais de Informação Tecnológica Industrial tais como a do PADCT, coordenada pelo IBICT/CNPq (através do Núcleo Regional de Informação Tecnológica do Estado de São Paulo - NRI e do Núcleo Especializado de Informação em Normas Técnicas - INTec), Sebrae e outras.

Como estratégias de atuação, busca por meio de seus produtos e serviços, a solução adequada para problemas tecnológicos existentes no mercado, com a oferta de:

- informação referencial: indicação de capacitação técnica do IPT; identificação de fornecedores de matérias-primas, produtos e equipamentos; informação sobre norma técnica; informação sobre o acervo de livros, publicações seriadas e periódicos, incluindo as publicações do IPT e outras obras bibliográficas; indicação de outras fontes de informação;
- pesquisas bibliográficas de natureza científica-tecnológica-industrial, com base no acervo de normas técnicas, catálogos de produtos industriais, livros, publicações seriadas, periódicos, na produção técnica do IPT e nas bases de dados de outros fornecedores;

- localização e recuperação de documentos nacionais e estrangeiros;

- elaboração de respostas técnicas, envolvendo bibliografia e consulta junto ao corpo técnico do IPT, para resolução de dúvidas referentes a processos industriais, uso de matérias-primas, características técnicas de produtos industriais;

- outros serviços e produtos, tais como assessoria técnica e orientação na concepção e gerenciamento de sistemas de informação para a área científica e tecnológica; informação para prospecção e monitoração tecnológica; implementação da atividade de extensão tecnológica no IPT; venda de publicações do IPT e de normas ABNT; cursos de sistemas de informação sobre tecnologia industrial básica, gestão estratégica do conhecimento, entre outros.

A experiência do Citec diante das diferentes linhas de atuação mencionadas e voltadas para o apoio ao desenvolvimento do setor produtivo no Brasil trouxeram consigo a necessidade de repensar o design de sua forma de gestão da informação para atender às demandas emergentes e o estabelecimento de novas parcerias e compartilhamentos.

Deste modo, o Citec voltou-se para diagnósticos, planejamento e avaliação de aspectos considerados de atenção primária ao estabelecimento de novas diretrizes que subsidiem a modernização das formas de gestão da informação para atender às demandas em cenários caracterizados por mudanças. São eles:

- identificação dos tipos predominantes de sua clientela;

- a pertinência e representatividade da informação demandada pelos clientes identificados;
- a oferta de serviços e produtos de informação necessária para responder a esta demanda, como os serviços da área de inteligência competitiva;

- a caracterização e a definição de políticas para aquisição de recursos informacionais.

Com a implantação dessa nova estrutura, o aumento da competitividade do Citec/IPT no mercado está se dando mediante a:

- potencialização do trabalho em equipe;

- crescimento da sinergia interequipes;

- capacitação e valorização dos recursos humanos disponíveis;

- potencialização do uso de equipamentos e softwares;

- racionalização do uso do espaço físico;

- reestudo da política de aquisição de informação e material bibliográfico;

- melhoria no índice de receitas/despesas do Citec;

- desenvolvimento de novas metodologias, visando ao aprimoramento e potencialização dos serviços oferecidos;

- aprimoramento da gestão do fluxo de informação tecnológica no Instituto;

- desenvolvimento de novos mercados e/ou parceiros.

Está sendo implementada nova visão da gestão da informação sob enfoque de modernos princípios envolvendo qualidade, competitividade e produtividade, aliados ao uso dos recursos das novas tecnologias, buscando fazer do Citec uma organização baseada na informação e no conhecimento para a sua efetiva contribuição ao desenvolvimento científico e tecnológico do IPT e do país. 


\section{MUDANÇA COMPORTAMENTAL DA EQUIPE DE PROFISSIONAIS DO CITEC/IPT}

O Citec/IPT está buscando solução para uma situação crítica, ou seja, a mudança da postura dos profissionais especializados em análise da informação, que estão sendo preparados para saber, com a maior clareza possível, quais são as informações úteis para a empresa aumentar sua produtividade, reduzir seus custos e desenvolver novos produtos e serviços.

Uma das grandes vantagens competitivas que o Citec/IPT tem em relação a outros concorrentes na prestação de serviços de informação é que as informações são validadas pelo corpo técnico do IPT.

Como visão de futuro quanto aos mercados interno e externo, pretende-se transformar efetivamente o CITEC em um centro de informação tecnológica voltado às atividades desenvolvidas pelas áreas técnicas do IPT, mediante duas ações básicas:

- monitorar os setores e fornecer informações que indiquem possibilidades de negócio;

- apoiar o desenvolvimento dos projetos mediante levantamento de informações sobre o estado da técnica.

Quanto ao mercado interno, pretendese ampliar e dinamizar o fluxo interno de informação entre o Citec e as unidades técnicas do IPT e organizar toda a base de conhecimento do IPT.

Quanto ao mercado externo, pretende-se ampliar e aprimorar o atendimento às micro, pequenas e médias empresas, mediante a oferta de serviços, tais como respostas técnicas, extensão tecnológica, levantamento de fornecedores, normas técnicas informação tecnológica. Para as grandes empresas, serviços como os de atualização de acervo de normas técnicas, oferta de pesquisa bibliográfica, inteligência competitiva e outros.
Parcerias e articulações com outras instituições e empresas usuárias e provedoras de informação são metas prioritárias para a implantação efetiva de um serviço de inteligência competitiva, visto que esse serviço é composto por um conjunto de ações sistematizadas de busca, de análise e de disseminação das informações úteis aos tomadores de decisão das instituições em geral.

Alguns fatores constituem objeto de atenção por parte da gestão do Citec:

- a questão da necessidade do compartilhamento de recursos de informação, na qual se faz necessário que o gerente desses sistemas apresente competências envolvendo a flexibilização, conhecimento da tecnologia da informação e a ampla identificação de fontes e canais de informação confiáveis e econômicos;

- a aquisição e/ou construção de fontes de informação que formem um acervo representativo com base na caracterização do comportamento da clientela quanto às demandas para uso de informação tecnológica no Citec, bem como propicie o emprego mais efetivo dos investimentos feitos recentemente no que diz respeito à nova infra-estrutura de instalações físicas e de equipamentos, possibilitando o uso de tecnologias para transmissão/recepção eletrônica de documentos;

- delineamento da política de aquisição e de compartilhamento de recursos informacionais, o que implica a necessidade de caracterizar o que adquirir em função das demandas informacionais; onde e como adquirir as informações necessárias para 0 atendimento a estas demandas, considerando-se a aquisição como um processo de agregar itens previamente selecionados a uma coleção, objetivando a maximização da utilização de recursos.

Para atender à clientela no que diz respeito à necessidade de dominar 0 processo de acompanhamento da evolução do ambiente concorrencial da empresa, o Citec/IPT deve estar apto a, em tempo hábil, recuperar, tratar e difundir informações que apontem mudanças, ocupando um espaço no mercado com a oferta de informações precisas e com valor agregado e que subsidiem as atividades de prospecção e monitoramento ativo do ambiente competitivo das empresas em suas necessidades de conhecimento.

A equipe atual de profissionais de informação no Citec/IPT, apesar de experiente e familiarizada nas diversas áreas de atuação do IPT, ainda carece de treinamento técnico para seu aperfeiçoamento, capacitação, atualização e valorização da informação.

Estes profissionais têm de reciclar seu conhecimento e adequar seu perfil às novas necessidades que se apresentam, pois, em um cenário de globalização e intensa competitividade, no qual as empresas passam por transformações organizacionais e tecnológicas em ritmo acelerado, atingirão vantagens competitivas aquelas organizações que puderem contar com agentes pró-ativos que administrem e recuperem informações voltadas às demandas do setor produtivo, oferecendo produtos e serviços de informação adequados às necessidades dos setores público e privado.

Para que isso ocorra, constitui nossa preocupação a busca de recursos humanos, financeiros e materiais para treinamento, capacitação e complementação da equipe.

Constituem quesitos fundamentais para uma mudança na cultura organizacional os seguintes indicadores de atuação:

- administração de biblioteca e arquivo - organização, preservação e oferta de serviços de acervos documentários, bibliográficos e não bibliográficos;

- documentação - formação, organização, análise, armazenamento, comunicação e avaliação de acervo documental especializado e memória organizacional;

- gestão da informação - planejamento, condução e avaliação dos processos de utilização dos recursos de informação necessários para incrementar a efetividade da organização; 
- gestão da tecnologia de informação - implementação e avaliação dos processos de adoção e adaptação das tecnologias da informação para modernizar e otimizar a gestão dos recursos e dos serviços de informação;

- gestão dos processos de comunicação: implementação e avaliação dos processos orientados à otimização da dinâmica comunicacional da organização;

- gestão do conhecimento - investigação dos processos de geração, transferência e aproveitamento do conhecimento em função do incremento da inteligência organizacional;

- engenharia do conhecimento - investigação e desenvolvimento de soluções apropriadas para a otimização das funções de representação, armazenamento, processamento e recuperação do conhecimento.
Conclui-se que, com recursos planejados e disponíveis, a busca e o atendimento à demanda de informações possam ser realizados sem necessidade de manter acervos bibliográficos com gama variada de informações, podendo se adotar uma nova plataforma de sistema de informação tecnológica baseada na identificação, localização, tratamento, busca e disponibilidade de informações pertinentes, independentemente de sua localização física. O que somente será possível mediante o surgimento de uma cultura organizacional aberta, participativa e democrática voltada para o próximo milênio.

\section{The competitive intelligence and the area of technological information at Instituto de Pesquisas Tecnológicas do Estado de São Paulo S. A. (Institute for Technological Research of São Paulo State)}

\begin{abstract}
This paper presents an appraisal of the change of paradigm which is being implemented in the present management of the Technological Information Center (Citec) of the Institute for Technological Researches of São Paulo State (IPT-SP). It is an attempt to display the performance of the information professional as an agent of change and the forms which can interfere in the organization culture, within the IPT new strategy, sharing risks of and benefits from the technological development in the productive, private and public sectors.
\end{abstract}

\section{Key words}

Technological information; Performance of the information professional; Professional as agent of changes; Organization culture.

\section{BIBLIOGRAFIA}

1. AUN, M.P. Capacitação de recursos humanos na área de informação tecnológica. Ciência da Informação, Brasília, v.25, n.1, p.43-46, 1996.

2. BARBOSA, R.R. Monitoração ambiental: uma visão interdisciplinar. Revista de Administração, São Paulo, v.32, v.4, p.42-53, out/dez, 1997.

3. RAMOS, P.A B. A gestão na organização de unidades de informação. Ciência da Informação, Brasília, v.25, n.1, p.15-25, jan/abr. 1996.

4. SANTOS JÚNIOR, J.N. Planejamento de serviços de ICT. Ciência da Informação, Brasília, v.25, n.1, p.47-51, 1996.

5. SANTOS, R.N.M. dos. Sistemas de informações estratégicas para a vitalidade da empresa. Ciência da Informação, v.25, n.1, p.12-14, jan/abr, 1994.

6. VIEIRA, Anna da S. Conhecimento como recurso estratégico empresarial. Ciência da Informação, Brasília, v.22, n.2, p.99-101, maio/ago.1993.
Sonia Maria Akiko Wada Tomimori

Bibliotecária e diretora técnica do Citec IPT, com Diplome d'Etude Approfundue de l'Universite de Droit d'Economie et des Sciences d'Aix Marseille III - France. 